\title{
Alliance to penetrate mysteries of the Earth
}

\author{
Gianpaolo Bellini ${ }^{1}$, Paolo Strolin ${ }^{2}$, Hiroyuki K.M. Tanaka ${ }^{3,}$ \\ ${ }^{1}$ Istituto Nazionale di Fisica Nucleare (INFN), Sezione di Milano, Milan, Italy \\ ${ }^{2}$ Università di Napoli Federico II, Dipartimento di Fisica, Naples, Italy \\ ${ }^{3}$ Earthquake Research Institute, The University of Tokyo, Tokyo, Japan
}

\section{Article history}

Received June 15, 2016; accepted October 5, 2016.

Subject classification:

Muography, Geo-neutrino, Earth, Volcano, Imaging.

\begin{abstract}
Muography and geoneutrinos, as applied to the investigation of Earth science topics, have developed during nearly the same timeframe. The idea of muography was first proposed in 1955 by E.P. George, a physicist who attempted to measure the areal density of the rock overburden of a tunnel underneath the Snowy Mountain Hydro-Electric Scheme in Australia. Thirteen years later, L. Alvarez first performed mugraphy in order to map out the internal structure of the Chephren's pyramid. With regard to geoneutrinos, George Gamow gave the first suggestion in a letter to F. Reines in 1953, two years prior to E.P. George's experiment. Enthusiastic about the idea in this letter, thirteen years later G. Eder discussed the potential of the "remarkable abundance of radioactive elements within the Earth". In the first decade of the 21st century, both the first muographic image of a volcano and the first measurement of geo-neutrinos respectively took place in 2006 and 2005. By encouraging the synergy of expertise in Earth science and particle physics, these new methods of studying previously invisible phenomenon within the Earth are continuing to improve as tools to solve Earth science challenges.
\end{abstract}

\section{Introduction}

The depths of the Earth have always stimulated the natural curiosity of the human species. For a long time they have remained inaccessible to knowledge, giving rise to beliefs and to visions typical of Science Fiction from its beginnings with Journey to the Center of the Earth by Jules Verne (1864). In particular, from the origins of human culture the "inner fire of the Earth" (Box 1) has always been present in myths, religion, philosophy and science, with beliefs and questions. This and other mysteries of the Earth are now in the realm of science, attempting to "see".

On November 14, 2014, the Japanese edition of the New York Times (Japan Times) was released with an article title: Italy, Japan promote cooperation in Earth Sciences using Physics. It refers to the signature of agreements for "allying" forces and expertise in investigating the depths of the Earth and the interior of volcanoes using new possibilities offered by elementary particles, namely "muons" and "neutrinos". The agreements involve the Italian National Institutes of Nuclear Physics (INFN) and of Geophysics and Volcanology (INGV), and the Earthquake Research Institute (ERI) of the University of Tokyo.

The alliance object of the agreement is twofold. It unites Italy and Japan, both subject to earthquakes and volcanic eruptions and at the vanguard in these researches. In addition, it unites Earth science and elementary particle physics in the study of phenomena that affect the entire population. It is so found again the spirit of "unity of science", which is at the origin of our culture and that tends to get lost with the inevitable spread apart in the specializations.

The signature occurred at the conclusion of a Workshop (Muographers 2014) hosted by the Italian Embassy in Tokyo, in which Italian and Japanese scientists have discussed the potential of the "muography" of the interior of volcanoes and of "geo-neutrinos". The latter are carriers of information on the heat that is released into the depths of the Earth. These are new scientific challenges that require new synergies.

Science is exploring the cosmos, but also the interior of the Earth is still full of mysteries. What is the source of energy (i.e. heat) that feeds the "inner fire of the Earth"? Is it only "primordial" heat, emanating from what remains of the Earth's energy dowry at the times of her formation, more than 4 billion years ago? Can geo-neutrinos tell something about it?

The internal heat of the Earth feeds the convective motions of the fluid matter in the Earth mantle below the crust, as the boiler circulates the water in a domestic radiator plant. It thus influences the "plate 


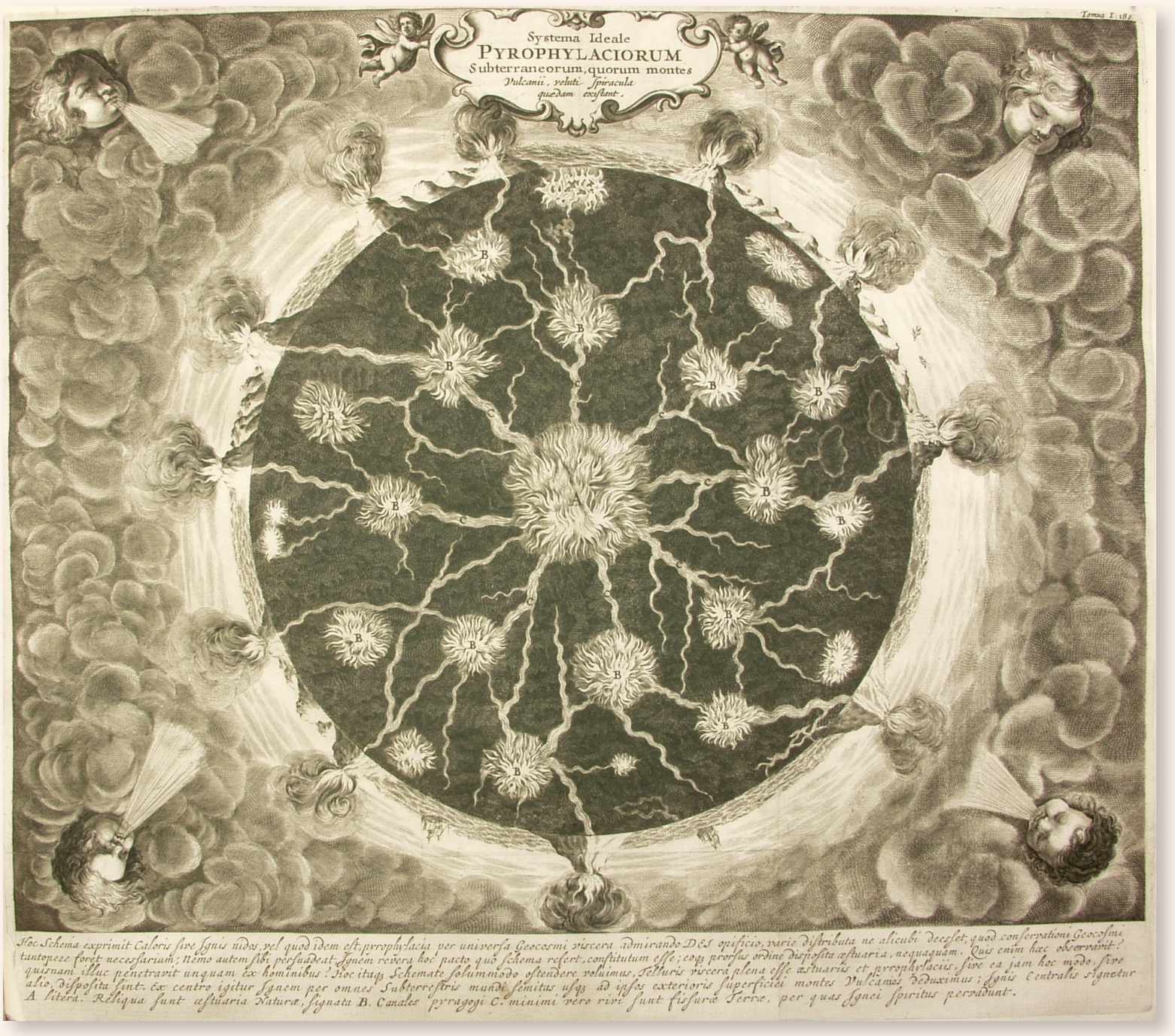

Figure 1. Athanasius Kircher, Mundus subterraneus (1664): Systema ideale pyro-phylaciorum subterraneorum, quorum montes vulcanii, veluti spiracula quaedam existant (Conceptual system of underground fire channels, of which the volcano mountains are like breathers). Photo: Klaus-Peter Kelber, University of Würzburg.

\section{Box 1. The inner fire of the Earth}

The idea of an "inner fire of the Earth" is already present in ancient Greek mythology, in which the underground world of the dead (Hades) had five rivers: Styx, Lethe, Cocytus, Acheron and Pyriphlegethon ("fireflaming").

Plato (about 425-348 BC) connects the volcanism to Pyriphlegethon: "after making many coils about the earth it plunges into Tartarus at a deeper level. This is that Pyriphlegethon, as the stream is called, which throws up jets of fire in all sorts of places" (Plato, Phaedo, 32-113b).

The Tartarus quoted by Plato is the deepest place

tectonics", with the consequent earthquakes, and the volcanic activities. Moreover, the eruptions depend on the pathways that magma and superheated material find to exit. Such pathways in the internal structure of volcanoes are mysteries hardly unaccessible to conventional techniques. of the damned by Zeus, "as far beneath Hades as heaven is about earth" (Homer, Iliad, VIII, 13).

The inner fire of the Earth enters Science with Athanasius Kircher (1602-1680), a few decades later than Galileo Galilei (1564-1642). His eclectic genius ranged from egyptology, geology, medicine and more. Observations on volcanoes made him to postulate the existence of a "fire" inside the Earth, which feeds the volcanism through a number of channels and of fiery intermediate chambers. The "mountains volcanoes" are considered "breathers" of the inner fire of the Earth. The figure comes from his work Mundus subterraneus (1664).

Questions are thus practical and not just speculative. Although the energy balance of the Earth is dominated by the heat absorbed from the Sun (about $99.97 \%$ ), the heat from its interior provides "driving force" to (sometimes devastating) phenomena that affect our lives. Let's see what muons and geo-neutrinos can do for us. 


\section{Muography of volcanoes}

Muons (denoted by the greek character $\mu$ ) are brothers of the electrons, but with a mass about 200 times larger. High energy muons are continuously produced in the interactions of very high energy particles from the cosmos (the so-called cosmic rays) with the particles of the Earth's upper atmosphere.

In "radiography" the transmission of X-rays in crossing the human body displays the "shadows" of the internal organs of greater thickness and/or density (for example, bones), and the presence of cavities (for example, dental caries). High-energy muons have a weaker interaction with matter and a strong penetration power, but similar properties in transmission. They can be used to explore through "muographies" internal structures on sizes much larger than those of the human body.

The idea of using muons to reveal internal structures otherwise inaccessible was first applied by Luis Alvarez around 1970 in archaeology for the search of unknown burial chambers in the Chafren's pyramid in Egypt (Box 2). The muography was performed by detecting muons having gone through the pyramid, projecting back their trajectories and studying their transmission.

But if muons could be used to study the pyramids, why not for volcanoes? Obviously in this case the amount of matter to be crossed is much higher: among those produced by cosmic rays only those of higher energy are capable of that, and they are which are more rare. This is how was born the use of muography to obtain "images" of the internal structure of the top part of volcanoes, highlighting for example the presence of low density areas or ducts. This ability to provide relatively direct and accurate images is certainly a step forward in techniques for the study of volcanic phenomena. Practically, since cosmic rays invest the Earth from all directions, there are also quasi horizontal muons and they can be exploited to perform muographies of structures which emerge from the Earth's surface, such as volcano edifices, by tracing back through them the muon trajectories as shown in Figure 3.

The muography was gradually developed. Twentyfive years after Alvarez, the paper reporting about an experimental investigation carried out in Japan by Kanetada Nagamine is concluded as follows: "it was made clear that nearly horizontal cosmic-ray muons can be used to explore the inner-structure of a gigantic geophysical substance, such as the top region of a volcano" [Nagamine et al. 1995].

The first important experimental proof in this field was given in 2007 by the muography of the summit of Mt. Asama in Japan [Tanaka et al. 2007]. The muography was carried out by one of the present authors (Hiroyuki Tanaka) in a collaboration with Kimio Niwa, taking advantage for muon detection of the developments of the

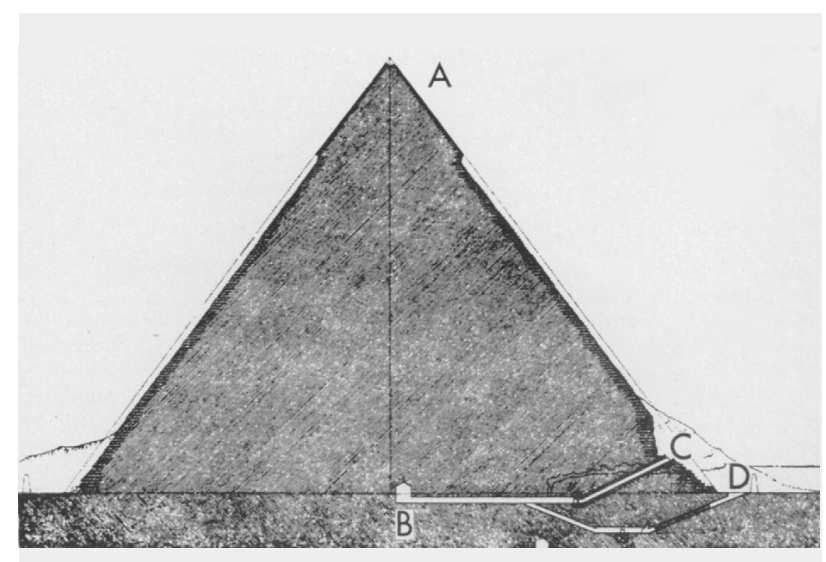

Figure 2. Elevation cross-section of the Chefren's pyramid in Giza. The pyramid height is about 140 meters. The muon detector was located in the Belzoni chamber (B) and was observing muons coming from the top through a rock thickness of the order of 100 meters. From Alvarez et al. [1970].

\section{Box 2. From rock overburden to muography}

The idea of exploiting cosmic-ray muons was first applied about 60 years ago by E.P. George, to evaluate the rock overburden of a tunnel of the Snowy Mountain Hydro-Electric Scheme in Australia through measuring the reduction of the muon flux in the transmission through the rock. The experimental apparatus was consisting of particle detectors called Geiger counters, providing an angular resolution inadequate to "image" any structure inside the rock overburden.

This technique was upgraded to "muography" by Luis Alvarez (1968 Nobel Prize for discoveries in particle physics) with the famous search of a hidden chamber in the Chefren's pyramid at Giza. The famous paper published on Science in 1970 says: "The principal novelty of the proposed cosmic-ray detectors involved their ability to measure the angles of arrival of penetrating cosmic-ray muons with great precision, over a large sensitive area $[\ldots]$ it was not until the invention of 'spark chambers' with digital read-out features that such a use could be considered as a real possibility" [Alvarez et al. 1970].

Luis Alvarez wondered why the Chefren's pyramid had only one burial chamber (the so-called Belzoni chamber) even though the nearby pyramid of his father Cheops had a more complex inner structure, including a King's chamber and a Queen's chamber. His calculations were showing that a hidden chamber would be "seen" by cosmic-ray muons observed in a spark chamber detector located in the Belzoni chamber, as a tooth cavity is seen as a black spot in a X-ray image.

In 1966, he started a joint project between United States of America and United Arab Republic. Although no hidden chamber was found, his pioneering work gave rise to the application of muography in various fields. 


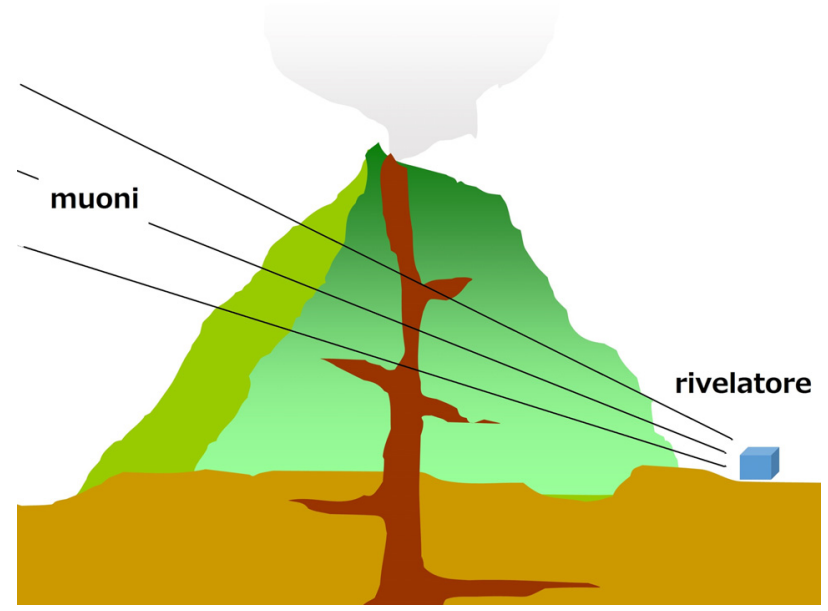

Figure 3. In muography, the experimental apparatus detects muons transmitted through the volcano.

"nuclear photographic emulsion" technique carried out for the OPERA neutrino experiment in the Gran Sasso underground laboratory.

Muography cannot provide images of the deep structure beneath the volcano (such as a deep magma chamber) but only of a relatively shallow region of the volcano edifice. Therefore it cannot give information about "when" an eruption might occur, but rather information useful to understand "how" it could develop. In particular, muographic images can give evidence of low-density magma pathways underneath a solidified magma deposit.

The muography of the Satsuma-Iwojima volcano performed by one of the present authors (Tanaka) provides an illustration of its advantages for studies of eruption dynamics. This volcano continuously discharges large amounts of gasses without significant magma emission. The muographic image shown on the left side of Figure 4 has revealed a low-density region at the uppermost point of the magma conduit, in accordance with a convection model predicting the presence of degassing magma at this location. In the convection model of K. Kazahaya, H. Shinohara and G. Saito [1994], which is shown on the right side of Figure 4, the magma conduit (cylindrical body) is connected to a deep magma chamber. The magma is pushed upwards by a gas component contained in it. In the upper part of the duct the gas is released and comes out from the volcano. The magma, no longer pushed by the gaseous component, re-sinks and therefore does not obstruct the conduit. A continuous supply of new magma pumped up by the gas that it contains ensures that the degassed magma is replaced and the cycle continues.

The same group has investigated other volcanoes in Japan. Here, we also mention the studies on the La Soufrière of Guadeloupe in the French Lesser Antilles by Dominique Gibert with the DIAPHANE collaboration [Lesparre et al. 2012].

Experiments are under way to assess the potentialities of muography for volcano investigations, and make progress in its development in order to extend the field of utilization. This requires the development of a new generation of detectors with improved performance. Schematically, this proceeds in two directions: one is the study of the time-development of eruptions in volcanoes where the rock thickness penetrated by muons remains limited to about half a kilometer. The necessary higher speed in accumulating data requires that a larger muon flux is intercepted and this in turn requires

$\begin{array}{lll}0.0 & 1.0 & 2.0(\mathrm{~km})\end{array}$

(m)
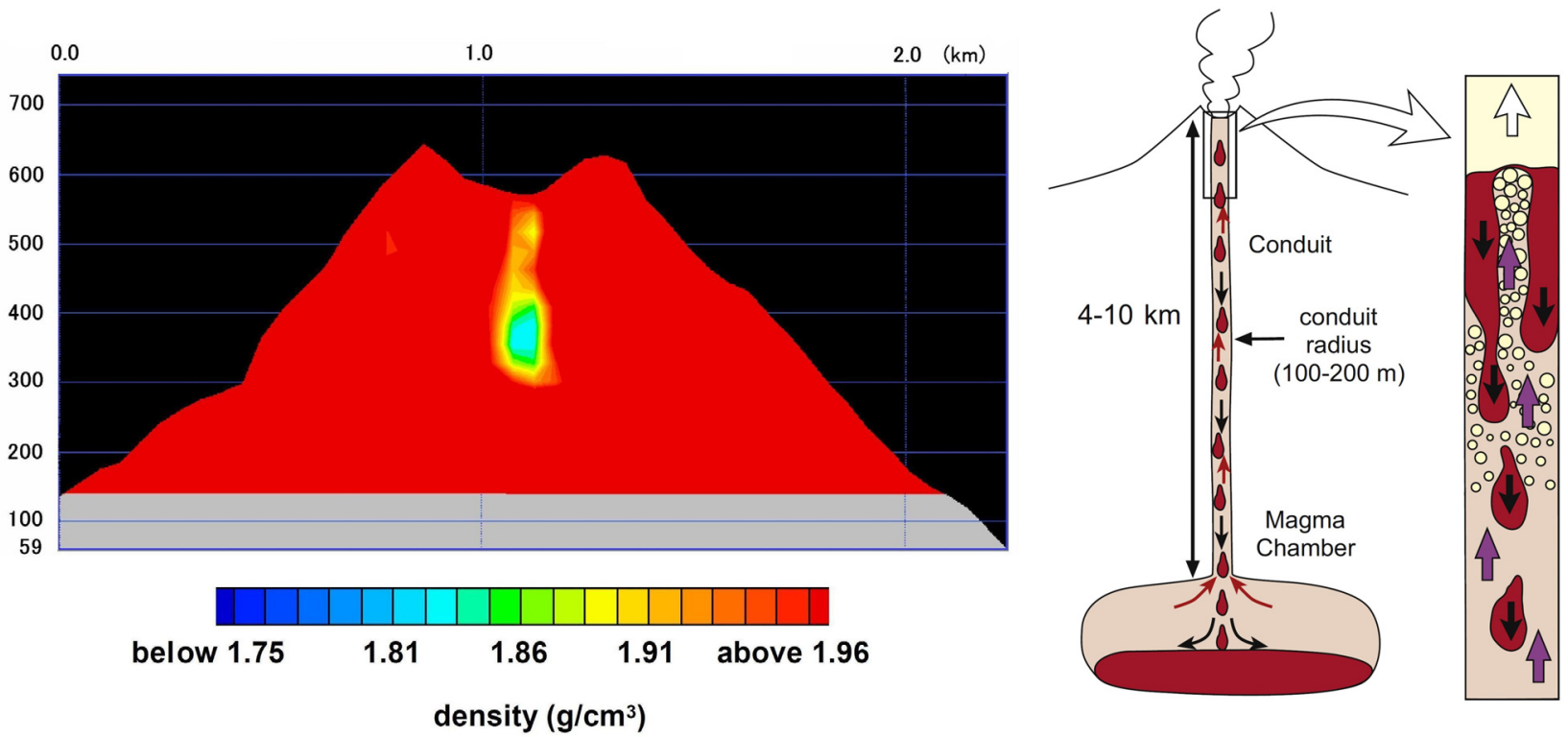

Figure 4. Left: Muographic image of the Satsuma-Iwojima volcano. A large, shallow depth, low-density region is visible beneath the crater floor, indicating degassing magma with its high proportion of bubbles. Right: Model of magma convection in a conduit at shallow depth. From Shinohara and Tanaka [2012]. 
an increase in the area covered by the experimental apparatus that detects muons (so far of the order of $1 \mathrm{msq}$ ). An additional aim is to improve the definition of the muographic images by a more precise tracing back of the muon trajectories through the volcano.

Another direction is the extension of the investigation to volcanoes that require muons penetrating through thicker layers of rock, therefore of higher energy. Their flow is fading with the energy (as we said muons of energy are also the rarest), and false signals caused by spurious effects (the "background") gradually become dominant. The situation becomes like when listening a faint voice in a noisy room; therefore in addition to a larger area, a very substantial reduction of such a background becomes essential. Test measurements have been performed at the volcano Puy de Dôme (France) with muons crossing a rock thicknesses of 1-1.5 kilometers of rock and detecting muons by incomplete prototypes of those foreseen by the MU-RAY and TOMUVOL collaborations led by Giulio Saracino (University of Naples "Federico II", and INFN) and by Cristina Cârloganu (French National Center for Scientific Research - CNRS), respectively. The analysis showed, with both prototypes, that the background is too high. This result is consistent with other indications and shows that an additional effort is required to reduce spurious effects. The development of new generation of muon detectors with wider area and new tools for background reduction is under way.

The diffusion of muography also requires developments in the treatment, understanding and interpretation of experimental carried out by an international community. The collaboration between MU-RAY and TOMUVOL quoted above is a step in this direction.

Altogether, the future of volcano muography will critically depend of a coherent and synergic effort of a strong international community of elementary particle physicists and geophysicists, in order to fully exploit its potential and turn it from a pioneering research field into a widely used tool for geophysical investigations.

We finally point out that the progress in volcano muography has stimulated applications to other fields. An example is a NASA project for the muography of asteroids in the solar system with size up to about $1 \mathrm{~km}$. It is based on the study of the transmission of muons produced in their superficial layers by the impact of high-energy particles coming from the cosmos.

\section{Geoneutrinos}

Everybody knows that a great amount of heat flows from the center of the Earth, through its surface, into space; but perhaps not everybody knows that a relevant fraction of this heath is due to the presence of

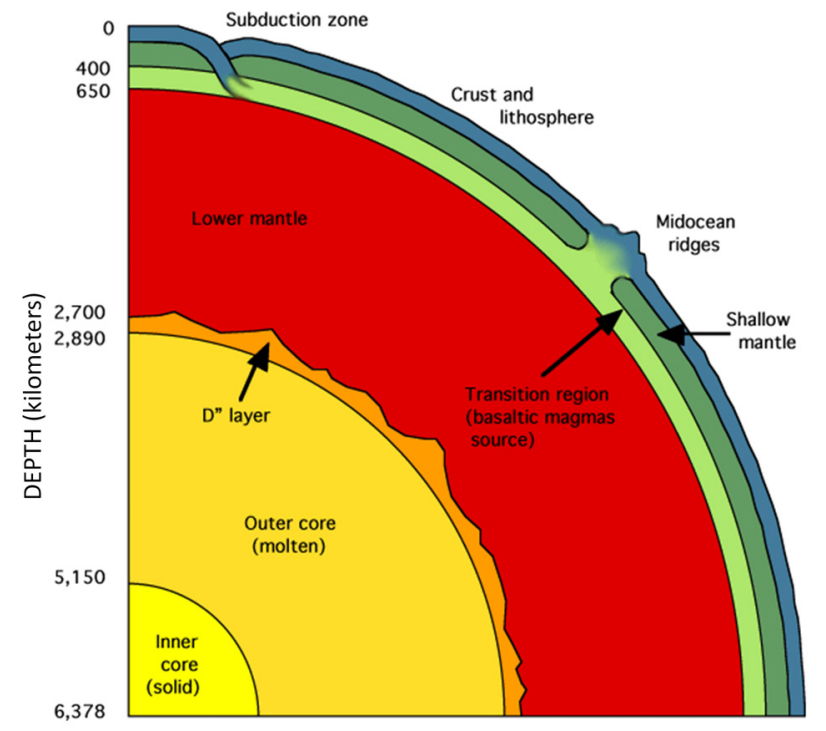

Figure 5. The inner structure of the Earth.

natural radio-nuclides in the Earth interior. The uranium and thorium and their daughter nuclei, in addition to the potassium, are distributed in the depths of our planet; they decay into other nuclei producing a known amount of heat. But how do we know that these radioactive nuclei are present in the Earth?

Such knowledge is possible thanks to tiny, chargeless particles: the neutrinos. The neutrinos and their antimatter counterparts, the anti-neutrinos, can cross large amounts of matter, very rarely interacting with it, remaining undisturbed. Therefore neutrinos are remarkably useful for probing regions that otherwise would be impossible to reach. A good example is offered by the Sun. While individual photons produced in the Sun take about 100,000 years to escape from the Sun's core, due to continuous absorptions and re-emissions during their travel through the solar matter, the neutrinos take just a few seconds to escape.

It is a lucky circumstance that the radioactive decays emit also anti-neutrinos, which, traveling to the surface, bring information on the amount and distribution of the radioactive elements in the depth of the Earth; by detecting anti-neutrinos, it is possible to estimate what fraction of the total terrestrial heat is produced by radioactive decays.

These anti-neutrinos are called geo-neutrinos and their study has opened a new era in the investigation of the structure of our planet.

As shown in Figure 5, our planet is an onion with five shells. The center of the Earth is an inner core, whose size, close to the Moon dimensions, consists basically of iron and nickel; the inner core is solid thanks to a very high pressure, despite the $\sim 5700 \mathrm{~K}$ of temperature. The inner core is surrounded by an outer core, having the same composition, but in a liquid state: its motion 
gives rise to the "geo-dynamo". An important shell, solid but viscose, $\sim 3100 \mathrm{~km}$ thick, surrounds the outer core: it is the lower mantle, composed of siliceous, magnesium and iron, where high temperature gradients produce important material movements (convective movements), which drive volcanic activities and displacements of tectonic plates. Finally, after the relatively thin ( $\sim 250 \mathrm{~km}$ of thickness) upper mantle, the very thin crust is the land on which we work, we walk and we live; it is divided into oceanic crust (younger and only $\sim 10 \mathrm{~km}$ thick) and continental crust (30-70 km thick).

Probably the reader is now wondering how geologists can obtain all these information. The methods used are essentially based upon the study of seismic waves and chemical analyses. The seismic waves, through their propagation, speed and behavior, provide information on the density of the crossed terrestrial regions and on their so-called phase, liquid or solid (for instance the sussultatory waves do not propagate in the liquids).

Geochemistry investigations are based upon the analysis of rock samples, extracted directly from the crust surface by drill-holes, and of mantle rocks, lifted by tectonic movements and volcanism, even if they can be altered during the transportation. The drill holes, which reach a maximum depth of $12 \mathrm{~km}$ in Russia, are useful for studying the crust composition, but not the mantle. Some information can be obtained by assuming that the chemical composition of our planet is the same as the chondritic meteorites' that landed on Earth, supporting the reasonable hypothesis that the solar system probably derives from a single primordial body. In particular the chondritic meteorites contain small spheres, the chondrites, $\sim 4.6$ billion years old (approximately the life time of the Earth), which experienced a very fast cooling and have then maintained unchanged their composition ever since.

The evaluation of the total surface heath flux of the Earth follows various approaches and thus presents a large range of values. Currently, geologists are shifting to a method based on obtaining the temperature gradient below the crust surface via 40,000 or so holes of different depths across the Earth, mostly drilled by oil-exploration firms. This method converges on $42 \pm 2$ billions of Watt (Terawatt) for the total heat flow.

Geo-neutrinos, like all types of neutrinos, are very elusive particles and capturing them is an extremely challenging task. Only two experiments have succeeded in studying the geo-neutrinos: KamLAND, located at the Kamiokande-Mozumi mine in Japan, and Borexino, at the Gran Sasso Laboratory, beneath the Apennines in central Italy. Both experiments use as detecting material several cubic meters of a liquid called scintillator that emits a faint light when a neutrino interacts with it. The total scintillator volume in Borexino is 300 meter cubes and in KamLAND 1000 meter cubes. Despite the large volumes the rate of detection of geo-neutrinos is very low: only one every seven weeks for Borexino and about three times more for KamLAND. Just for a comparison, the solar neutrinos, which strike the Earth at a rate of 60 billions per centimeter square per second, produce in Borexino about 45 detectable interactions per day.

A major problem in detecting geo-neutrinos is dealing with the interactions produced in the scintillator by the decay products of natural radio-nuclides and by the radiations from the cosmic space: the so-called background. Therefore, the detectors have to be installed in underground locations below an overburden of rock, which absorbs the particles produced by the cosmic rays that strike the atmosphere components. In addition, the detectors have to be shielded from fake signals originated from the radioactive nuclides of everything around them: the rocks surrounding the underground hall, the materials present in the location as well as the air. But the toughest challenge is reducing the intrinsic radioactivity of the components of the detector itself and especially of the scintillator.

Borexino has been designed to detect interactions from the solar neutrinos at energies much lower than the geo-neutrinos, hence, a very low radioactivity level of the scintillator was mandatory.

Borexino has successfully reached a very high and unprecedented radio-purity. For KamLAND, radio-purity requirements were not so compelling since it was designed to detect the antineutrinos from nuclear reactors.

However the antineutrinos from reactors are themselves another source of fake events in measuring antineutrinos. This is a particular problem for KamLAND, which has to contend with an antineutrino flux seven time as high as Borexino's, because of the higher density and closer proximity of nuclear reactors in Japan rather then in Italy (although in Japan reactors were off for a certain period of time after the Fukushima accident). Fortunately antineutrinos from reactors are spread over an energy range partially higher than the geo-neutrinos.

Figure 6 shows the geo-neutrinos signals obtained by the two experiments (Borexino on the left [Bellini et al. 2013], and KamLAND on the right [Gando et al. 2013]) with the various contributions of the background.

The discovery of geo-neutrinos opens remarkable new possibilities for exploring the properties of the Earth's interior, helping to answer the following questions concerning its composition, origin and development. We know that radioactive nuclei are present in the Earth's crust. For reasons of chemical affinity they may be present also in the mantle (not in the core). The first question concerns the actual presence of radioactive 

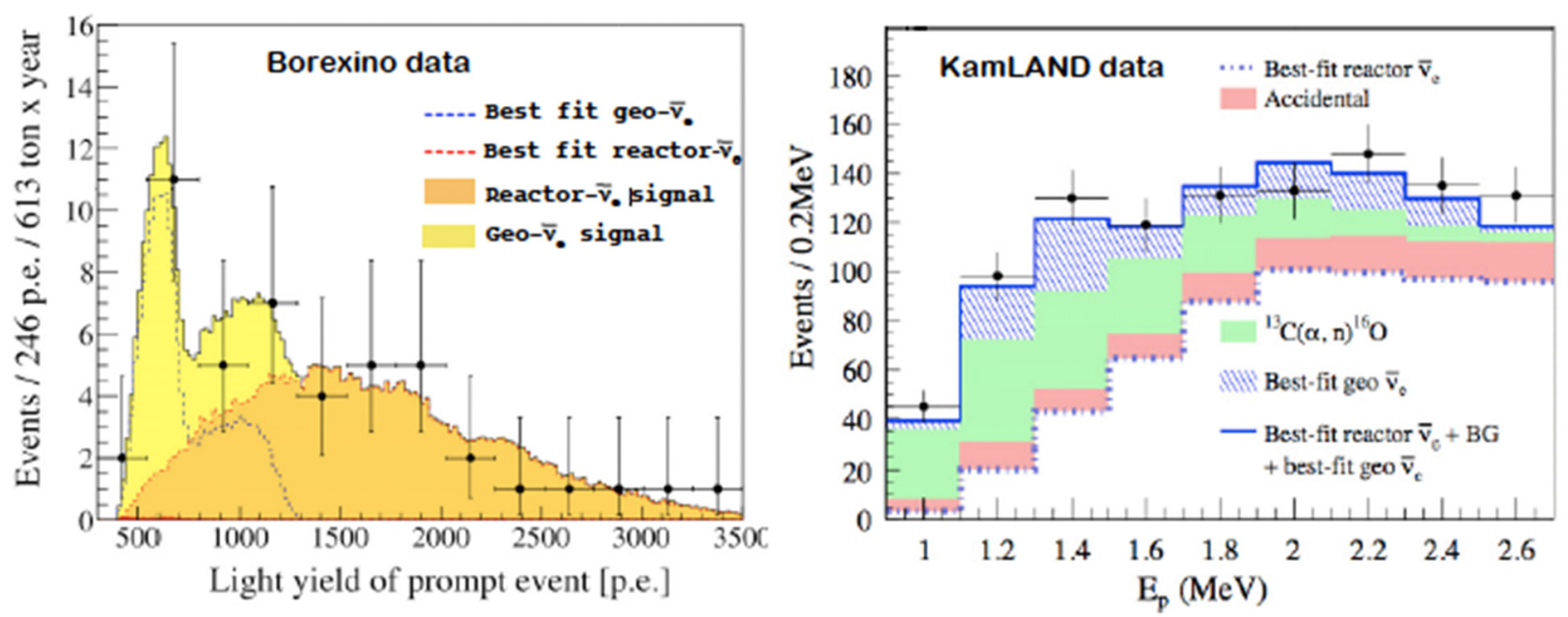

Figure 6. Borexino (left) e KamLAND (right) data. The geo-neutrino signal is the yellow area for Borexino and the blue area for KamLAND. In Borexino the inner radioactive background is negligible; in KamLAND corresponds to the red and green areas. The areas, ocher for Borexino e white for KamLAND, are the signals of the antineutrinos from nuclear reactors.

nuclei also in mantle and the contribution of their decays to the global terrestrial heat.

Second, in order to better understand the origin of the Earth and of the solar system, it is important to know the ratio of the amounts of uranium and thorium present in terrestrial matter and to see if it agrees with that found in chondritic meteorites.

Finally, one tries to discriminate the validity of the Earth's models (called BSE, or bulk silicate Earth) developed by geophysicists, which follow three different approaches: a) cosmo-chemical, which takes as a basis for the chemical composition of terrestrial matter that of meteorites; b) geo-chemical, which also is based upon the chemical composition of chondritic meteorites, but in addition takes account of the composition of the rocks from the mantle; c) geo-dynamic, which takes into account the energy of convective motions in the mantle.

To answer the question on the radioactivity in the mantle, in addition to the measurement of the total flux of geo-neutrinos, one must know that from the crust around the site where the detector is installed, as well as from the crust at a distance. For this purpose, one analyzes the rocks from the detector site and one studies the geological structure of the crust knowing the rocks that compose it. Putting together the data Borexino and those of KamLAND, a positive indication of mantle radioactivity has been obtained, but with a still relevant uncertainty.

The contribution of radioactive decays to terrestrial heat can be inferred from the radioactivity of the mantle and the contribution of the crust. Unfortunately, the uncertainties on the distribution of radioactive elements in the mantle - homogeneous distribution or concentration at the close of the core - produce a degree of uncertainty still large in this evaluation. The ratio of the signals coming respectively from uranium and thorium in chondritic meteorites is 3 . The same ratio is 2.5 according to the study of the distribution of geo-neutrino energy made by Borexino. Given the experimental uncertainty it is compatible with the chondritic ratio, as it is the measurement made by KamLAND, although suffering from a larger experimental uncertainty. Finally the results obtained to date seem to favor the biochemical approach between models BSE.

For an upgrading of the results, more statistics are undoubtedly needed. Borexino and KamLAND are proceeding to take data during further several years. However, in the meantime, other experiments have been launched, in particular $\mathrm{SNO}+$, installed underground at Sudbury (Canada), which soon will start taking the data and expects 28-30 geo-neutrino events per year. A very interesting experiment is HanoHano that will be installed under the sea of Hawaii. Since Hawaii are placed on a thorium-and-uranium-depleted oceanic crust, $70 \%$ of the signals will be produced in the mantle! Therefore, this experiment could lead to very important results. Unfortunately the cost and the complexity of the experiment have so far prevented its approval and funding.

Several detectors, as Borexino or KamLAND, spread out around the world could provide more information on the heat distribution in the mantle and also on the convective movements taking place in it, that is the tectonic plate movements and the volcanic activities. However, neutrino detectors require great effort and are significantly expensive.

\section{Concluding remarks}

The investigation into the basic features of the Earth's interior has been a particularly active area of research in the field of Earth science, and has been conducted over the 20th century by using "classical probes" such as seismic waves. Seismic tomography has revealed, 
e.g., that the Earth has a layered structure comprising of the core, mantle and crust.

Proceeding into the 21 st century, research may expand by utilizing "quantum probes" - namely cosmic-ray muons and geo-neutrinos - and unveil features otherwise inaccessible. Muography was born more than forty years ago with an application to archaeology and recently experienced a remarkable progress in volcanology, by exploiting experimental techniques developed for elementary particle physics. Geo-neutrinos have recently been discovered by underground experiments in Italy and Japan, designed for physics and astrophysics investigations related to elementary particles. The potential of such quantum probes as tools in Earth science is starting to be recognized.

About three millennia of human history after the Pyriphlegethon of ancient Greece, a large deal of mysteries about the Earth and its "inner fire" persists. Great discoveries may be pursuable by muography and geoneutrinos for surveys of the interior of the Earth. Both require experimental ingenuity and challenging dedicated projects, which can be made reality only through alliances between the communities of elementary particle physics and Earth science under a common vision of science.

The spirit of unity of science has characterized great scientists through millennia. The progress of science has required a specialization in scientific disciplines, however dominating only since as little as about one century. The new approach to science required to explore by quantum probes the interior of "our" Earth, with alliances among different fields of research, is thus coherent with the roots and the history of scientific culture.

N.B. An Italian version of this article has been previously published on Le Scienze, 564, August 2015.

\section{References}

Alvarez, L.W., J.A. Anderson, F. El Bedwei, J. Burkhard, A. Fakhry, A. Girgis, A. Goneid, F. Hassan, D. Iverson, G. Lynch, Z. Miligy, A.H. Moussa, MohammedSharkawi and L. Yazolino (1970). Search for Hidden Chambers in the Pyramids, Science, 167, 832-839.

Bellini, G., et al. (Borexino collaboration) (2013). Measurement of geo-neutrinos from 1353 days of Borexino, Phys. Lett. B, 722, 295-300.

Gando, A., et al. (KamLAND collaboration) (2013). Reactor on-off antineutrino measurement with KamLAND, Phys. Rev. D, 88, 033001.

Kazahaya, K., H. Shinohara and G. Saito (1994). Excessive degassing of Izu-Oshima volcano: magma convection in a conduit, B. Volcanol., 56, 207-216.

Lesparre, N., et al. (DIAPHANE collaboration) (2012).
Density muon radiography of La Soufrière of Guadeloupe volcano: comparison with geological, electrical resistivity and gravity data, Geophys. J. Int., 190, 1008; doi:10.1111/j.1365-246X.2012.05546.x.

Nagamine, K., M. Iwasaki, K. Shimomura and K. Ishida (1995). Method of probing inner-structure of geophysical substance with the horizontal cosmic-ray muons and possible application to volcanic eruption prediction, Nucl. Instrum. Meth. A, 356, 585-595; doi:10.1016/0168-9002(94)01169-9.

Shinohara, H., and H.K.M. Tanaka (2012). Conduit magma convection of a rhyolitic magma: Constraints from cosmic-ray muon radiography of Iwodake, Satsuma-Iwojima volcano, Japan, Earth Planet. Sc. Lett., 349-350, 87-97.

Tanaka, H.K.M., T. Nakano, S. Takahashi, J. Yoshida, M. Takeo, J. Oikawa, T. Ohminato, Y. Aoki, E. Koyama, H. Tsuji and K. Niwa (2007). High resolution imaging in the inhomogeneous crust with cosmic-ray muon radiography: The density structure below the volcanic crater floor of Mt. Asama, Japan, Earth Planet. Sc. Lett., 263, 104-113; doi:10.1016/j. eps1.2007.09.001.

\footnotetext{
${ }^{\star}$ Corresponding author: Hiroyuki K.M. Tanaka, Earthquake Research Institute, The University of Tokyo, Tokyo, Japan; email: ht@eri.u-tokyo.ac.jp.

C 2017 by the Istituto Nazionale di Geofisica e Vulcanologia. All rights reserved.
} 